\title{
Machine Learning for Photovoltaic Systems Condition Monitoring: A Review
}

\author{
$1^{\text {st }}$ Tarek Berghout \\ University of Batna2 \\ Department of Industrial Engineering \\ and Manufacturing \\ Batna, Algeria \\ t.berghout@unive-batna2.dz \\ $4^{\text {th }}$ Siniša Djurović \\ The University of Manchester \\ Department of Electrical and \\ Electronic Engineering \\ Manchester, UK \\ Sinisa.Durovic@manchester.ac.uk
}

\author{
$2^{\text {nd }}$ Mohamed Benbouzid \\ University of Brest \\ UMR CNRS 6027 IRDL \\ Shanghai Maritime University \\ Brest, France \\ Mohamed.Benbouzid@univ-brest.fr \\ $5^{\text {th }}$ Leïla-Hayet Mouss \\ University of Batna2 \\ Department of Industrial Engineering \\ and Manufacturing \\ Batna, Algeria \\ h.mouss@univ-batna2.dz
}

\author{
$3^{\text {rd }}$ Xiandong Ma \\ Lancaster University \\ Engineering Department \\ Lancaster, UK \\ xiandong.ma@lancaster.ac.uk
}

\begin{abstract}
Condition Monitoring of photovoltaic systems plays an important role in maintenance interventions due to its ability to solve problems of loss of energy production revenue. Nowadays, machine learning-based failure diagnosis is becoming increasingly growing as an alternative to various difficult physical-based interpretations and the main pile foundation for condition monitoring. As a result, several methods with different learning paradigms (e.g. deep learning, transfer learning, reinforcement learning, ensemble learning, etc.) have been used to address different condition monitoring issues. Therefore, the aim of this paper is at least, to shed light on the most relevant work that has been done so far in the field of photovoltaic systems machine learning-based condition monitoring.
\end{abstract}

Keywords-Photovoltaic systems, condition monitoring, machine learning.

\section{INTRODUCTION}

Renewable energies have important results for energy consumption in many sectors, including public and industry. Their climate-friendly energy transformation translates into the attraction of power generation companies from around the world [1]. Statistics from "World Energy Data" [2] explain that energy consumption with renewable energy resources reaches $23.6 \%$ of world energy consumption. Among the many renewable energy resources, solar energy consumption occupies $11.44 \%$ of the total amount of renewable energy expenditure with future potential increase. However, satisfying energy consumption needs and ensuring high quality distribution totally depends on a reliable condition monitoring system capable of real-time assessment of health status while providing necessary information on maintenance planning [3]. Accuracy of a condition monitoring system is itself based on a well-constructed virtual model capable of simulating the behavior of the actual studied system [4]. Most of the literature works indicate that a set of rational physical interpretations (thermal modeling, electrical modeling, etc.) will certainly lead to a very powerful model in case of a lower depth level of the treated problem [5]. The depth of the problem lies in the complexity of the system that may include multiple parameters such as number of components, nature of interactions, and external effects [5]. As the complexity reasonably increased, data-driven solution will be the only available modeling paradigm. Among data-driven methods, while taking into account new varieties of advanced sensor technologies, especially in the Industry 4.0 era, machine learning (ML) theories have become one of the main way of large problems treatment [1]. As a result, in the field of health condition monitoring of photovoltaic (PV) systems, several ML approaches have been studied in-depth. Several training paradigms ranging from hybrid to deep learning has been discussed.

Some recent reviews provide very insightful information on the use of ML in assessing the health of PV systems. Indeed, work was carried out by K. M. Sundaram et al. [6] provides a lot of important information on the application of deep learning tools for several industrial systems, including PV panels. It deals with deep architectures such as autoencoders, convolutional neural networks (CNN), long short-term memory (LSTM), deep stacking network (DSN), deep belief networks, and the extension to generative adversarial networks (GANs). The work of S. Zhao et al. [3] analyze ML methods depending on the type of application, including remaining useful life (RUL) prediction, anomaly detection, and degradation analysis. G. Spagnuolo et al. [4] provide an overview of a set of condition monitoring tools used for accurate monitoring, diagnosis, and prognosis of PV systems.

The above-mentioned works focus on the use of machine learning tools for PV condition monitoring and provides a general interpretation of their application nature. However they mostly did not move towards the study of feature collection (e.g. ordinary sensors, wireless, and unmanned aerial vehicles (UAV)) and extraction techniques (e.g. thermal image processing, signal processing, and data exploration with ML). In addition, many details about number of failures and operating conditions are missing. Architectures and extensions to real operating conditions different from maximum power point tracking (MPPT) are not fully covered. To the best of the authors knowledge, these woks represents some scarcity in presenting works that have been done in the presence of very massive imbalanced dynamic data, which can be found in real application. In this context, this focused paper is proposed to review PV systems machine leaning-based condition monitoring works covering the aforementioned missing details, while suggesting ways of likely futures opportunities on PV systems health monitoring.

This paper is organized as follow: Section II presents used ML models with respect to their feature extraction and collection techniques, as well as problems of failures modes types and numbers and data dimensionality. Section III provides important conclusions and suggests ways forward. 


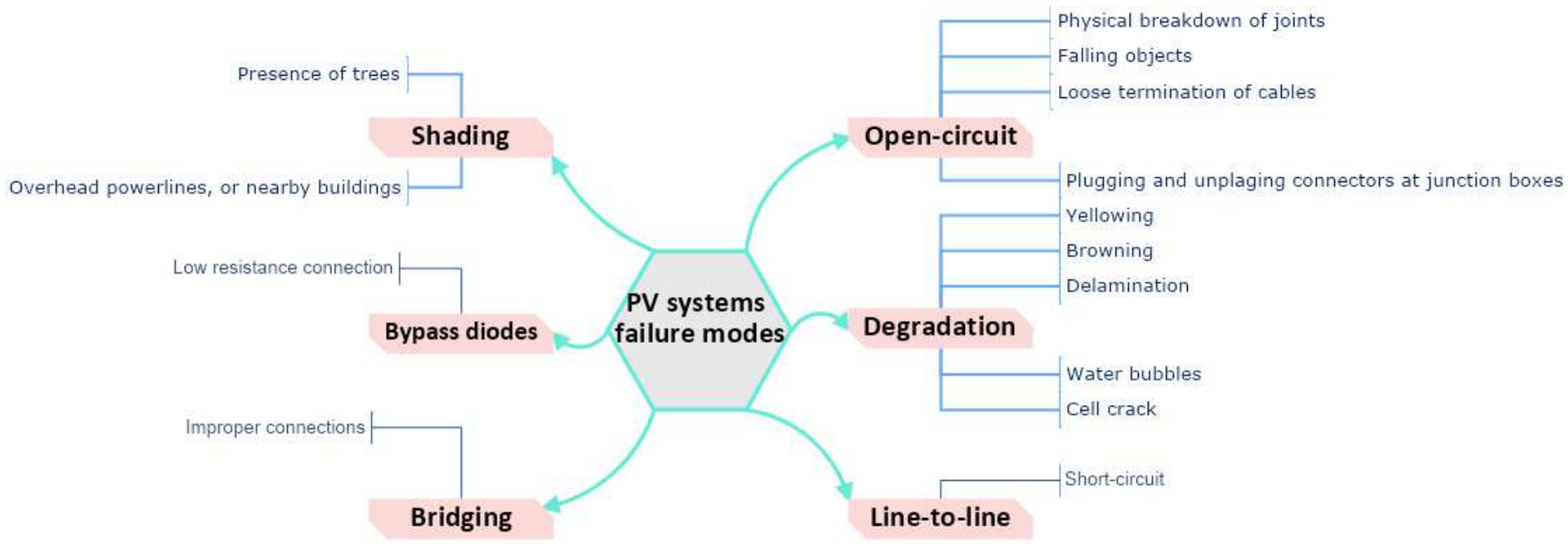

Fig. 1. Common failure types of PV systems.

\section{MACHINE LEARNING FOR PV SYSTEMS CONDITION MONITORING}

Health prediction consisted of evaluating the operating state of the system to detect common failures of photovoltaic systems. Common types of faults in PV systems can be classified into 6 different groups. Fig. 1 elucidates the main possible causes of their dysfunction.

Condition monitoring of PV systems with ML tools generally involves two main steps: i) selection of important features and extraction of parameters and ii) training process for failures classification. Features extraction and selection techniques depend on the amount of training data (e.g. Bigdata) and the nature of the collected samples (e.g. time series, thermographic images, etc.). In this context, Fig. 2 highlights these steps in a single flowchart.

In learning process, the prediction algorithm choice depends on the complexity of the solution model reconstruction (see J. Schmidhuber [5], §3). Therefore, this section will list different ML approaches according to three main classes: i) conventional ML tools including hybrid and ensemble learning paradigms; ii) advanced deep learning models; iii) recent knowledge-driven methods such as GANs and TL. A focus will also be made on on the number of treated failures and their detection nature. Fig. 3 is an illustration of the proposed classification studied in this paper.

\section{A. Conventional Machine Learning Models}

Unlike deep learning algorithms, conventional ML paradigm does not focus much more on learning from representations than on universal approximation [7]. Their main objective is to achieve greater accuracy by producing the best loss error. In this context, E. Garoudja et al. [4] trained a probabilistic neural network (PNN) using currentvoltage (I-V) analysis on the DC converter side to distinguish faulty and normal operating conditions. The processed data were retrieved from a simulation model after identification of the parameters of the grid-connected PV module. Four failure modes, around short circuits, with different number of strings and disconnection of strings from PV array were considered. H. Momeni et al. [8] proposed an ML approach for PV systems failure diagnosis.

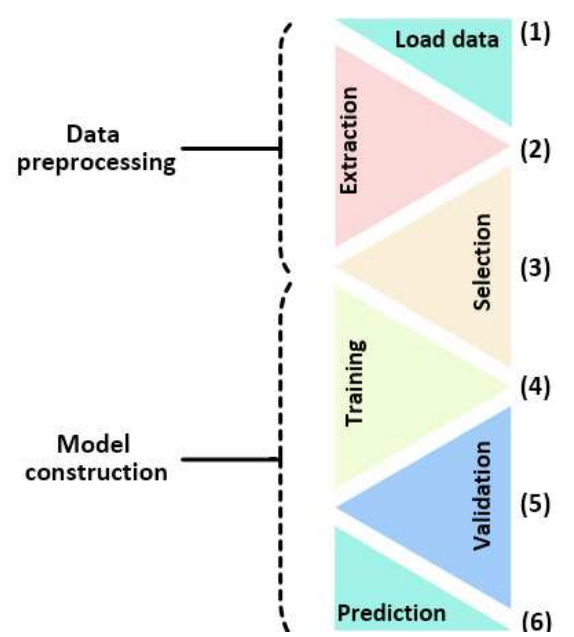

Fig. 2. Model reconstruction steps.

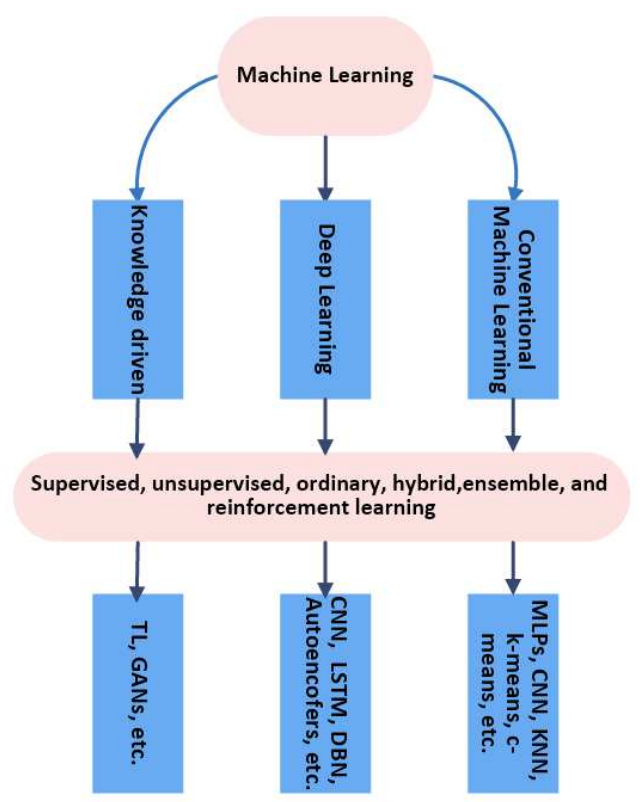

Fig. 3. ML tools classification.

Diagnosis algorithms were essentially built in a semisupervised learning process. A graph-based learning approach (GBSSL) was used to generate labels for new 
unseen samples based on some analysis of a set of labeled samples. Two types of failures linked to different shortcircuit cases were studied using the same information methodology (I-V). L. Maaløe et al. [9] generate a dataset with several types of failures modes (10 modes) from a real life-based simulation model. Then, Bayes theorem (BT) was used as a clustering model to distinguish between different operating behaviors depending on the I-V characteristic monitoring. Clustering results were fed into a classifier based on stochastic gradient ascent methods to achieve the approximation process. A. E. Lazzaretti et al. [10] used a dynamic adaptive recursive (DARL) linear model to detect failure modes (normal, short circuit, degradation, shading) in PV systems under various operating conditions. A photovoltaic power plant simulator was developed and was able of generating the required training and test data. The simulation model was carried out on the basis of a real existing PV system to latter facilitate validation. Failures were intentionally injected into the model to produce enough samples under various operating conditions. K. Dhibi et al. [11] employed a reduced-kernel random forest (RKRF) algorithm for failure detection and classification in a gridconnected PV system. They used principal component analysis (PCA) to reduce the dimensionality of the training samples as well as they involved K-nearest neighbors (KNN) clustering to reduce the number of observations. A PV simulation model was involved to evaluate the classification process of the RF algorithm. A set of $9 \mathrm{I}-\mathrm{V}$ sensors was placed in different positions to be able to collect the needed healthy patterns to detect 5 different faulty modes of different components (sensor, inverter, grid-connection, PV panels, etc.). J. M. Huang et al. [12] optimized the extreme learning machine (ELM) algorithm using bee colony (BC) algorithm to perform accurate PV systems failure diagnosis by studying dust as the main environmental variable. A. Eskandari et al. [13] also follows the same above-mentioned path for PV systems failure detection but an overall learning architecture was used. It was a bit more complex than the previously mentioned work. Indeed, different algorithms were involved, including $\mathrm{KNN}$, support vector machine (SVM), and naive Bayes (NB) in a unique training process. A voting process will then lead to a decision. Two cases of healthy and faulty states were treated as a classification problem. In [14], the same path condition monitoring path has been followed but, unlike ensemble learning, a technique was adopted to choose the best classifier from three selected ones, namely SVM, NB, and logistic regression (LR).

Contrary to the above-mentioned works, entirely depending on the analysis of $\mathrm{I}-\mathrm{V} / \mathrm{P}-\mathrm{V}$ characteristic, $\mathrm{M}$. U. Ali [15] provides a different technique based on infrared thermographic images to determine hotspots in PV modules. Many feature extraction techniques such as RGB, texture, oriented gradient histogram (HOG), and local bit pattern (LBP) based on image processing were involved in the first step. After several tests with different datasets, SVM was proven to be able to accurately solve classification problems. Similar experiments have been reproduced by M. Dhimish [16] and show that discriminant classifiers (DC) leads to better accuracy than SVM and other conventional machine learning methods.

All in one, the aforementioned condition monitoring techniques mainly use the I-V/P-V characteristic analysis where data in the training process have been driven by simulation models inspired from real PV installations.
However, this context is clearly less complex and far from real-world PV systems applications handling a huge amount of different data types (I, V, thermographic images from installed cameras, environmental variables). Treatment of prediction problems in a more complex way is shown in the work of A. Eskandari et al. [13] where ensemble learning was used as it will provide accurate predictions. However, the issue of rich dynamic data is not fully covered, which could limit the current contributions to some level of lower depth problems than real world applications.

Table I summarizes main works considering PV systems condition monitoring using conventional machine learning paradigms.

\section{B. Advanced Deep Learning Techniques}

Deep learning algorithms entirely focus on feature representations. The more robust the representations are, the more accurate the learning process will be [17]. Within deep multiple layers of nonlinear abstractions, features space will be greatly improved and some important patterns will emerge, which will lead to a greater propagation of decision classes. As a result, even greater generalization will be gained. Accordingly, many deep learning frameworks have been proposed to treat the issue of dimensionality and representations learning. For instance, and unlike conventional manual feature extraction, A. Y. Appiah et al. [18] proposed a fully automatic feature extraction technique based on LSTM, which is highly capable of sequence-tosequence training due to its learning ability through time. In this study dealing with PV systems condition monitoring using data-driven methods, focus was on failure analysis using I-V information that were injected into a simulation model. Unlike previously mentioned works, a large number of failures types were adopted and classified onto two main categories, namely line-line failure (LLF) and hot spot failure (HSF). It is worth to mention that an amount of 2240 and 1961 different cases were simulated for both LLF and HSF, respectively.

TABLE I. CONVENTIONAL MAChine LeARning PARAdigms.

\begin{tabular}{|c|c|c|c|}
\hline References & Tools & Detection & Failures \\
\hline E. Garoudja et al. [4] & PNN & $\mathrm{I}-\mathrm{V}$ & 4 \\
\hline Momeni et al. [8] & GBSSL & $\mathrm{I}-\mathrm{V}$ & 2 \\
\hline L. Maaløe et al. [9] & BT & $\mathrm{I}-\mathrm{V}$ & 10 \\
\hline A. E. Lazzaretti et al. [10] & DARL & $\mathrm{I}-\mathrm{V}$ & 4 \\
\hline Dhibi et al. [11] & $\begin{array}{l}\text { RKRF } \\
\text { PCA } \\
\text { KNN }\end{array}$ & $\mathrm{I}-\mathrm{V}$ & 5 \\
\hline J. M. Huang et al. [12] & $\begin{array}{c}\text { ELM } \\
\text { BC } \\
\text { KNN }\end{array}$ & $\mathrm{I}-\mathrm{V}$ & 5 \\
\hline A. Eskandari et al. [13] & $\begin{array}{l}\text { SVM } \\
\text { NB }\end{array}$ & $\mathrm{I}-\mathrm{V}$ & 2 \\
\hline A. Eskandari et al [14] & $\begin{array}{c}\text { SVM, } \\
\text { NB } \\
\text { LR }\end{array}$ & $\mathrm{I}-\mathrm{V}$ & 2 \\
\hline M. U. Ali [15] & SVM & $\begin{array}{c}\text { Thermographic } \\
\text { images }\end{array}$ & 2 \\
\hline M. Dhimish [16] & $\mathrm{DC}$ & $\begin{array}{c}\text { Thermographic } \\
\text { images }\end{array}$ & 2 \\
\hline
\end{tabular}

In the meantime, 1866 cases of healthy states have been collected. X. Li et al. [19] investigated more complex failure classification problems based on real data obtained from large-scale PV farms. Drone technology was used to collect 
different surface images, classifying various forms of modulus failures, including dust, shading, encapsulant delamination, glass breakage, gridline corrosion, snail trails, and yellowing. According to the depth of big-data issues, deep CNN learning algorithms were adopted. In another work [20], both drone technology and machine learning were adopted for PV plants failure detection. In this case, a CNN was adopted for extracting appropriate training features, and instead of fine-tuning of the CNN itself, SVM was used to learn from the extracted patterns and for failure classification. W. Gao et al. [21], based on many learning parameters (i.e. I-V characteristics, solar irradiance, temperature) a deep CNN was built and reinforced by a residual gated recurrent unit (Res-GRU) to be able to perform both dynamic and adaptive learning under a higher level of nonlinear feature abstractions when classifying multiple types of PV systems failures. The designed networks allow for self-automatic extraction and less intervention when classifying not only single failure types (e.g. short circuit, partial shading, abnormal aging, etc.) but also hybrid types. R. Pierdicca et al. [22] used thermal images obtained through infrared sensor installed in a drone to train a mask region-based $\mathrm{CNN}$ algorithm for patterns recognition problem under PV condition monitoring criteria. In this context, the 3 considered failure modes were related to cells (one anomaly, not contiguous cells with anomalies, contiguous cells with anomalies).

Table II summarizes main works considering PV systems condition monitoring using deep learning paradigms.

\section{Extension to Knowledge-Guided Models}

Lack of descriptive patterns in training data is due to many issues such as difficulty in collecting data or lack of real failure mode models related to less down times cases. As a result, many methods have been proposed to generate additive hypotheses to augment training data or for existing generalization process. In recent literature, GAN and TL learning paradigms have proven their unique ability to extend network generalization by providing new hypotheses on similar learning samples.

GANs are learning networks developed by Goodfellow in 2015 [23]. Their main way is to train a generative model such as an autoencoder to generate real examples from fakes using a minimax two-player game approach. However, this time the generator is trained in a supervised way by associating a discriminator to classify these representations in the two preceding categories.

S. Lu et al. [24], [25] proposed a hybrid deep TL mechanism allowing domain adaptation with a CNN algorithm for DC arc failure diagnosis (PV system).

TABLE II. DEEP LEANING PARADIgMS

\begin{tabular}{lccc}
\hline \multicolumn{1}{c}{ References } & Tools & Detection & Failures \\
A. Y. Appiah et al. [18] & LSTM & I-V & 2 \\
X. Li et al. $[19]$ & CNN & $\begin{array}{c}\text { UAV Surface } \\
\text { images }\end{array}$ & 7 \\
X. Li et al. [20] & CNN & UAV Surface \\
images & 7 \\
W. Gao et al. [21] & SVM & I-V \\
R. Pierdicca et al. [22] & Res-GRU & $\begin{array}{c}\text { Solar irradiance } \\
\text { Temperature }\end{array}$ & 3 \\
\hline
\end{tabular}

First, the algorithm tries to learn representative examples from the learning samples on the source domain data. After that, a dummy generation process of new samples in the target domain is followed by the TL process using GANs. W. Tang et al. [26], under a prediction problem using limited number of electroluminescence images, augmented their data by combining GANs and traditional image processing techniques. After that, generated examples are fed into a CNN failure detection in PV modules. M. W et al. [27] also proposed the use of transfer learning approach to train a CNN for PV failure classification on two main datasets: electroluminescence images dataset in the source domain and infrared images dataset on the target domain.

It can be observed that knowledge-driven models by the use of different training paradigms such as TL and GANs are problems of an incomplete data learning process. Therefore, further knowledge must involve the extension of the generalization.

Table III summarizes main works considering PV systems condition monitoring using knowledge-driven paradigms.

\section{CONCLUSIONS AND WAYS FORWARD}

One can observe from the literature state of the art review that traditional machine learning tools with different paradigms (hybrid and ensemble) are generally used to investigate time series analysis. Driven samples most of the time are depends on conventional acquisitions systems that involves I-V/P-V curves analysis. Therefore it is almost rare to find such an application of traditional machine learning in image processing for condition monitoring of PV systems.

In the meantime, advanced deep learning techniques have been widely applied for different types of image processing including electroluminescent and infrared thermographic. Knowledge-driven models are generally used when training and testing data suffers from non-similarity in distributions besides lack of patterns and icomplete liste of labels.

Generally speaking these algorithms are used under similar criteria that inclouds: i) these algorithms treated the same PV systems condition monitoring issues (i.e. failure detection and diagnosis) under the same working conditions which always is set to the MPPT; ii) most of them are trying to detect healthy patterns from data obtained from simulation models; iii) treatment of data classes is almost the same (i.e. $\approx 3 \pm 2$ ), apart from some works such as H. Momeni et al. [8] where 10 failure modes were considered. In addition, traditional machine learning models generally treats I-V characteristics to reach their goal of best approximation. Meanwhile, deep networks are generally treating deeper problems with higher number of features including images.

TABLE III. KNOWLEDGE-DRIVEN PARADIGMS.

\begin{tabular}{|c|c|c|c|}
\hline References & Tools & Detection & Failures \\
\hline S. Lu et al. [24], & $\begin{array}{c}\text { CNN } \\
\text { TL } \\
\text { GAN } \\
\text { CNN }\end{array}$ & Only current (I) & 1 \\
\hline S. Lu et al. [25]. & $\begin{array}{c}\text { TL } \\
\text { GAN }\end{array}$ & Only current (I) & 1 \\
\hline W. Tang et al. [26] & $\begin{array}{l}\text { CNN } \\
\text { GAN }\end{array}$ & $\begin{array}{c}\text { Electroluminescence } \\
\text { images }\end{array}$ & 4 \\
\hline M. W. Akram et al. [27] & $\begin{array}{l}\mathrm{CNN} \\
\mathrm{TL}\end{array}$ & $\begin{array}{l}\text { Electroluminescence } \\
\text { and infrared images }\end{array}$ & 3 \\
\hline
\end{tabular}


More complex operating conditions were considered by A. Bakdi et al. [28], where both MPPT and intermediate PPT (IPPT) were considered resulting in 16 failure modes that have been intentionally injected in a simulation model inspired. However, initial row data was recorded in real time and is not generated from a probability distribution. There were collected from two real scenarios of healthy operation under MPPT and IPPT.

The recorded scenarios were extremely large dynamic environment that is able to produce a deep problem. Bakdi et al. [28] attempt to solve this problem by proposing the use a hybrid model that combines a set of ordinary ML tools namely principal component analysis (PCA), KullbackLeibler divergence (KDL), recursive smooth Kernel density estimation (KDE). These tools were respectively applied for dimensionality reduction, failure features extraction, and approximation. Better performances were achieved but the problem still needs a deeper evaluation of deep learning tools.

In this context, ways forward will be including deep learning models under similar complex criteria of higher dimensional data (MPPT and IPPT modes) for PV systems condition monitoring. Besides, one can provide more indepth conclusions by investigating more complex studies including both infrared images and sensors measurements in transfer learning scheme.

\section{REFERENCES}

[1] N. Khadka, A. Bista, B. Adhikari, A. Shrestha, D. Bista, and B. Adhikary, "Current Practices of Solar Photovoltaic Panel Cleaning System and Future Prospects of Machine Learning Implementation," IEEE Access, vol. 8, pp. 135948-135962, 2020, doi: 10.1109/ACCESS.2020.3011553.

[2] World energy data, "World total final consumption," 2020. https://www.worldenergydata.org/world-total-final-consumption/.

[3] S. Zhao and H. Wang, "Enabling Data-Driven Condition Monitoring of Power Electronic Systems With Artificial Intelligence: Concepts, Tools, and Developments," IEEE Power Electron. Mag., vol. 8, no. 1, pp. 18-27, Mar. 2021, doi: 10.1109/MPEL.2020.3047718.

[4] E. Garoudja, A. Chouder, K. Kara, and S. Silvestre, "An enhanced machine learning based approach for failures detection and diagnosis of PV systems," Energy Convers. Manag., vol. 151, no. September, pp. 496-513, 2017, doi: 10.1016/j.enconman.2017.09.019.

[5] J. Schmidhuber, "Deep Learning in neural networks: An overview," Neural Networks, vol. 61, pp. 85-117, 2015, doi: 10.1016/j.neunet.2014.09.003

[6] K. M. Sundaram, A. Hussain, P. Sanjeevikumar, J. B. Holm-Nielsen, V. K. Kaliappan, and B. K. Santhoshi, "Deep Learning for Fault Diagnostics in Bearings, Insulators, PV Panels, Power Lines, and Electric Vehicle Applications - The State-of-the-Art Approaches," IEEE Access, vol. 9, pp. 41246-41260, 2021, doi: 10.1109/ACCESS.2021.3064360.

[7] T. Berghout, L. H. Mouss, O. KadriI, and N. Hadjidj, "Regularized Length Changeable Extreme Learning Machine with Incremental Learning Enhancements for Remaining Useful Life Prediction of Aircraft Engines," in 020 1st International Conference on Communications, Control Systems and Signal Processing (CCSSP), May 2020, pp. 358-363, doi: 10.1109/CCSSP49278.2020.9151607.

[8] H. Momeni, N. Sadoogi, M. Farrokhifar, and H. F. Gharibeh, "Fault Diagnosis in Photovoltaic Arrays Using GBSSL Method and Proposing a Fault Correction System," IEEE Trans. Ind. Informatics, vol. 16, no. 8, pp. 5300-5308, 2020, doi: 10.1109/TII.2019.2908992.

[9] L. Maaløe, O. Winther, S. Spataru, and D. Sera, "Conditional monitoring in photovoltaic systems by semi-supervised machine learning," Energies, vol. 13, no. 3, pp. 1-14, 2020, doi: $10.3390 / \mathrm{en} 13030584$

[10] A. E. Lazzaretti et al., "A monitoring system for online fault detection and classification in photovoltaic plants," Sensors (Switzerland), vol. 20, no. 17, pp. 1-30, 2020, doi: 10.3390/s20174688.
[11] K. Dhibi et al., "Reduced Kernel Random Forest Technique for Fault Detection and Classification in Grid-Tied PV Systems," IEEE J. Photovoltaics, vol. 10, no. 6, pp. 1864-1871, 2020, doi: 10.1109/JPHOTOV.2020.3011068.

[12] J. M. Huang, R. J. Wai, and G. J. Yang, "Design of Hybrid Artificial Bee Colony Algorithm and Semi-Supervised Extreme Learning Machine for PV Fault Diagnoses by Considering Dust Impact," IEEE Trans. Power Electron., vol. 35, no. 7, pp. 7086-7099, 2020, doi: 10.1109/TPEL.2019.2956812.

[13] A. Eskandari, J. Milimonfared, and M. Aghaei, "Line-line fault detection and classification for photovoltaic systems using ensemble learning model based on I-V characteristics," Sol. Energy, vol. 211, no. July, pp. 354-365, 2020, doi: 10.1016/j.solener.2020.09.071.

[14] A. Eskandari, J. Milimonfared, and M. Aghaei, "Fault Detection and Classification for Photovoltaic Systems Based on Hierarchical Classification and Machine Learning Technique," IEEE Trans. Ind. Electron., vol. 0046, no. c, pp. 1-10, 2020, doi: 10.1109/TIE.2020.3047066.

[15] M. U. Ali, H. F. Khan, M. Masud, K. D. Kallu, and A. Zafar, "A machine learning framework to identify the hotspot in photovoltaic module using infrared thermography," Sol. Energy, vol. 208, no. July, pp. 643-651, 2020, doi: 10.1016/j.solener.2020.08.027.

[16] M. Dhimish, "Defining the best-fit machine learning classifier to early diagnose photovoltaic solar cells hot-spots," Case Stud. Therm. Eng., vol. 25, no. February, p. 100980, 2021, doi: 10.1016/j.csite.2021.100980

[17] T. Berghout, L. H. Mouss, O. Kadri, L. Saïdi, and M. Benbouzid, "Aircraft engines Remaining Useful Life prediction with an adaptive denoising online sequential Extreme Learning Machine," Eng. Appl. Artif. Intell., vol. 96, p. 103936, Nov. 2020, doi: 10.1016/j.engappai.2020.103936.

[18] A. Y. Appiah, X. Zhang, B. B. K. Ayawli, and F. Kyeremeh, "Long short-Term memory networks based automatic feature extraction for photovoltaic array fault diagnosis," IEEE Access, vol. 7, pp. 3008930101, 2019, doi: 10.1109/ACCESS.2019.2902949.

[19] X. Li, Q. Yang, Z. Lou, and W. Yan, "Deep Learning Based Module Defect Analysis for Large-Scale Photovoltaic Farms," IEEE Trans. Energy Convers., vol. 34, no. 1, pp. 520-529, 2019, doi: 10.1109/TEC.2018.2873358.

[20] X. Li, W. Li, Q. Yang, W. Yan, and A. Y. Zomaya, "An Unmanned Inspection System for Multiple Defects Detection in Photovoltaic Plants," IEEE J. Photovoltaics, vol. 10, no. 2, pp. 568-576, 2020, doi: 10.1109/JPHOTOV.2019.2955183.

[21] W. Gao and R. J. Wai, "A Novel Fault Identification Method for Photovoltaic Array via Convolutional Neural Network and Residual Gated Recurrent Unit," IEEE Access, vol. 8, pp. 159493-159510, 2020, doi: 10.1109/ACCESS.2020.3020296.

[22] R. Pierdicca, M. Paolanti, A. Felicetti, F. Piccinini, and P. Zingaretti, "Automatic Faults Detection of Photovoltaic Farms: solAIr, a Deep Learning-Based System for Thermal Images," Energies, vol. 13, no. 24, p. 6496, 2020, doi: 10.3390/en13246496.

[23] I. J. Goodfellow et al., "Generative Adversarial Networks," Jun. 2014, [Online]. Available: http://arxiv.org/abs/1406.2661.

[24] S. Lu, T. Sirojan, B. T. Phung, D. Zhang, and E. Ambikairajah, "DADCGAN: An Effective Methodology for DC Series Arc Fault Diagnosis in Photovoltaic Systems," IEEE Access, vol. 7, pp. 4583145840, 2019, doi: 10.1109/ACCESS.2019.2909267.

[25] S. Lu, R. Ma, T. Sirojan, B. T. Phung, and D. Zhang, "Lightweight transfer nets and adversarial data augmentation for photovoltaic series arc fault detection with limited fault data," Int. J. Electr. Power Energy Syst., vol. 130, no. April, p. 107035, 2021, doi: 10.1016/j.ijepes.2021.107035.

[26] W. Tang, Q. Yang, K. Xiong, and W. Yan, "Deep learning based automatic defect identification of photovoltaic module using electroluminescence images," Sol. Energy, vol. 201, no. November 2019, pp. 453-460, 2020, doi: 10.1016/j.solener.2020.03.049.

[27] M. W. Akram, G. Li, Y. Jin, X. Chen, C. Zhu, and A. Ahmad, "Automatic detection of photovoltaic module defects in infrared images with isolated and develop-model transfer deep learning," Sol. Energy, vol. 198, no. November 2019, pp. 175-186, 2020, doi: 10.1016/j.solener.2020.01.055

[28] A. Bakdi, W. Bounoua, A. Guichi, and S. Mekhilef, "Real-time fault detection in PV systems under MPPT using PMU and high-frequency multi-sensor data through online PCA-KDE-based multivariate KL divergence," Int. J. Electr. Power Energy Syst., vol. 125, no. July 2020, p. 106457, Feb. 2021, doi: 10.1016/j.ijepes.2020.106457. 